\title{
The tumor suppressor role of miR-4782-3p in hepatocellular carcinoma
}

\author{
WENTAO BO, YONG HU, XIELIN FENG, HUI ZHANG, LANG TIAN and AIXIANG LIU \\ Department of Hepatobiliary and Gastrointestinal Surgery, Sichuan Provincial Cancer Hospital, \\ Chengdu, Sichuan 610041, P.R. China
}

Received November 1, 2015; Accepted December 15, 2015

DOI: $10.3892 /$ or.2016.4568

\begin{abstract}
Hepatocellular carcinoma (HCC) is the second leading cause of cancer-related mortalities in China. Although advances have been made in treatments, the prognosis of HCC patients has not improved significantly. MicroRNAs (miRNA) play important roles in all stage of the progress of HCC. miR-4782-3p takes part in the pathogenesis of non-small cell lung cancer (NSCLC). However, the role of miR-4782-3p in HCC remains unknown. In the present study, we found that miR-4782-3p had low expression in HCC tissues. The low expression of miR-4782-3p indicated shorter survival of HCC patients. Moreover, the low expression of miR-4782-3p promoted HCC cells growth and inhibited cell apoptosis. We confirmed that USP14 was targeted by miR4782-3p in HCC cells.
\end{abstract}

\section{Introduction}

HCC accounts for 70 to $90 \%$ of primary liver cancer of the world, and is the third major cause of cancer-related mortality worldwide $(1,2)$. In China, HCC is the second leading cause of cancer-related mortalities $(3,4)$. Recently, progress has been made in HCC treatment; however, the prognosis of HCC patients has not improved significantly. There are so many challenges remaining. Moreover, it is difficult to diagnose $\mathrm{HCC}$ in the early stage, and many patients develop cancer recurrence after liver resection (5). Therefore, the development of novel therapeutic molecular targets is urgently needed.

MiRNAs play important roles in the pathogenesis of HCC (6-22). For example, miR-940 levels in HCC tissues were lower than normal liver tissues, lower miR-940 expression in HCC tissues significantly correlated with the reduced patient

Correspondence to: Dr Aixiang Liu, Department of Hepatobiliary and Gastrointestinal Surgery, Sichuan Provincial Cancer Hospital, 55 People's South Road 4th Section, Chengdu, Sichuan 610041, P.R. China

E-mail: aixiangliucancer@163.com

Key words: miR-4782-3p, cell growth, apoptosis, patient survival, USP14 survival rate, low level of miR-940 promoted HCC cell growth and inhibited cell apoptosis (23). Another study showed that miR-99a was an independent predictor for the prognosis of HCC patients (24).

A previous study showed that miR-4782-3p level in NSCLC tissues is lower than in normal lung tissues. High expression of miR-4782-3p indicated the favorable prognosis of NSCLC patients. The targeted genes were USP14, ZEB2 and XIAP (25). Another study showed that USP14 activation promoted tumor progression in HCC (26). Thus we considered that miR-4782-3p may also play an important role in HCC via USP14.

In this study, we assayed miR-4782-3p expression in HCC tissues and tested the function of miR-4782-3p in HCC cells. Our data elucidated the function of miR-4782-3p in HCC.

\section{Materials and methods}

Patients. Twenty-seven HCC specimens were collected from the Department of Hepatobiliary and Gastrointestinal Surgery, Sichuan Provincial Cancer Hospital (Chengdu, China). Tissue samples were immediately frozen in liquid nitrogen after isolation. Informed consent was obtained from each patient. The Ethics Commitment of Sichuan Provincial Cancer Hospital and the Ethic Commitment of Sichuan University approved this study. The senior pathologists of Sichuan Provincial Cancer Hospital evaluated the histological features of the specimens.

Cell culture. HCC cell lines HepG2 and SMMC-7721 and, L-02 cells were purchased from the American Type Culture Collection (ATCC; Rockville, MD, USA). Human HCC HepG2, SMMC-7721 and L-02 cells were maintained in Dulbecco's modified Eagle's medium (DMEM) with $10 \%$ fetal bovine serum (FBS; Invitrogen Corporation, Grand Island, NY, USA).

Detection of miR-4782-3p level in HCC tissues or cells. Total RNAs of HCC tissues and cells were extracted by TRIzol reagent (Invitrogen Corporation) according the manufacturer's instruction. The total RNAs was reverse-transcribed to cDNA by using All-in-One ${ }^{\mathrm{TM}}$ miRNA First-Strand cDNA Synthesis kit (Invitrogen Corporation). The primers were designed and synthesized by Shengong Company (Shengong, Shanghai, 

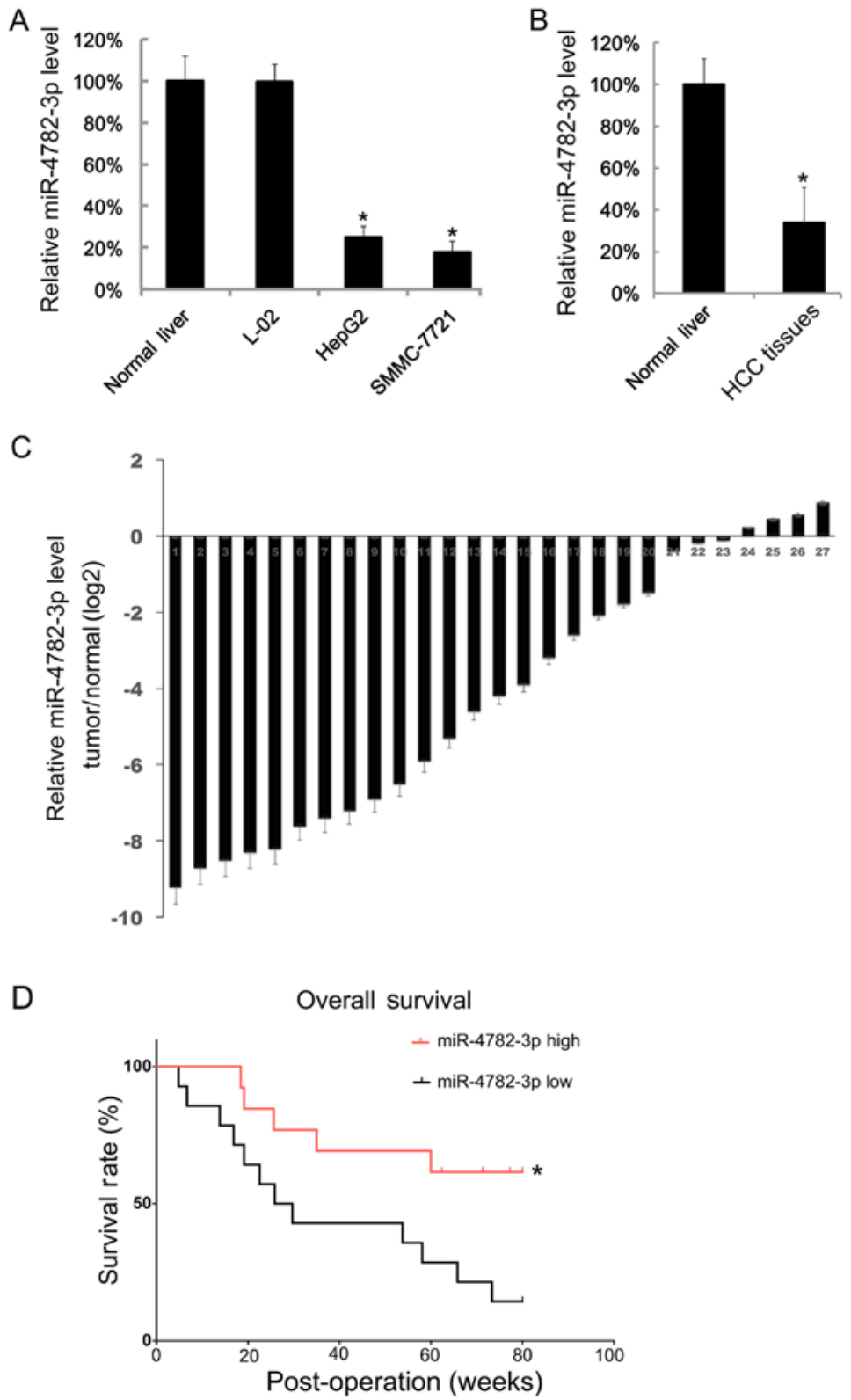

Figure 1. The lower expression of miR-4782-3p in HCC tissues and its' role in the survival of HCC. The expression of miR-4782-3p in normal liver tissues, and L-02, HepG2 and SMMC-7721 cells were revealed by qRT-PCR. U6 snRNA was used as a negative control (A). The level of miR-4782-3p in 27 pairs of human HCC tissues and matched adjacent normal liver tissues were tested by qRT-PCR, the mean level of 27 normal liver tissues were arbitrarily defined as $100 \%$ (B). After evaluation of the miR-4782-3p expression, data are shown as $\log 2$ of fold change of HCC tissues relative to adjacent normal liver tissues. U6 snRNA was used as negative control (C). The median miR-4782-3p expression value separated the 27 HCC patients into two groups: miR-4782-3p high level group and miR-4782-3p low level group. Kaplan-Meier survival test indicates that patients with higher miR-4782-3p levels had significantly longer survival (D). The qRT-PCR experiments were performed three times. Data are mean $\pm \mathrm{SD}$. ${ }^{*} \mathrm{P}<0.05$.

China). Real-time PCR assay was performed as described previously $(24,27)$.

Oligonucleotides and cell transfection. miR-4782-3p antisense oligonucleotides (miR-4782-3p ASO), miR-4782-3p mimics and negative control miRNA were purchased from RiboBio (Guangdong, China). miR-4782-3p ASO or miR-4782-3p mimics were transfected into cells by using Lipofectamine (Invitrogen, Shanghai, China), according to the manufacturer's instruction (27).

Cell proliferation by MTT analysis. HepG-2 and SMMC-7721 cells $\left(5 \times 10^{3} /\right.$ well $)$ were seeded into 96 -well plates. Then MTT experiments were performed as previously described $(28,29)$. Absorbance in each well was measured by using a microplate reader set at $570 \mathrm{~nm}$.

Apoptosis analysis. The apoptosis of HepG-2 and SMMC7721 cells transfected with miR-4782-3p ASO or miR-4782-3p mimics were analyzed by using PI/Annexin V staining and fluorescence-activated cell-sorting (FACS) flow cytometer (Biosciences, Beijing, China). Details in brief, HepG2 and SMMC-7721 cells were seeded into 12-well plates. Twelve hours after transfection, cells were cultured with serumdepleted medium for $12 \mathrm{~h}$. Then cells were suspended in binding buffer followed by staining with PI/Annexin V-FITC 
A

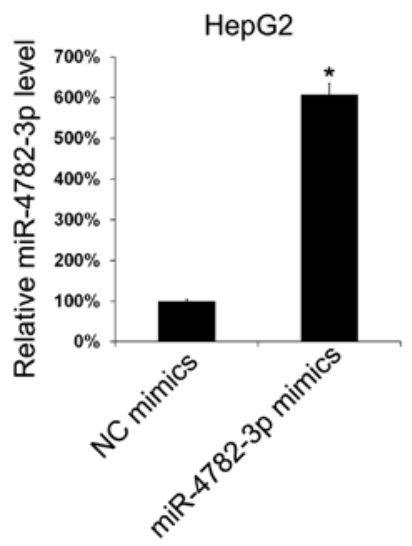

B

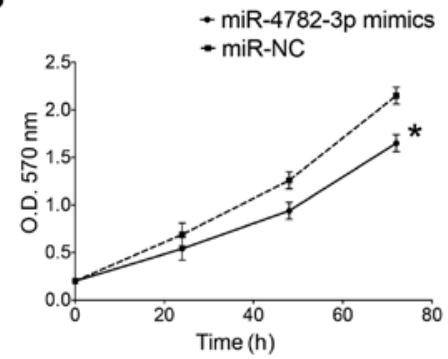

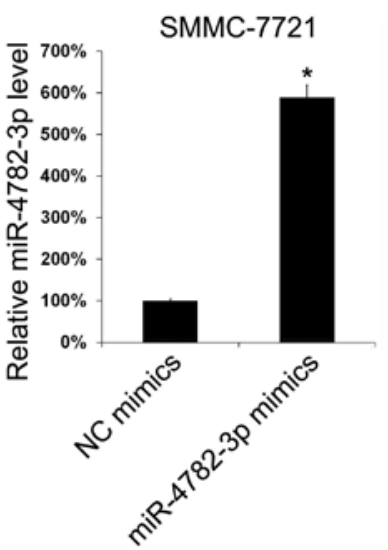

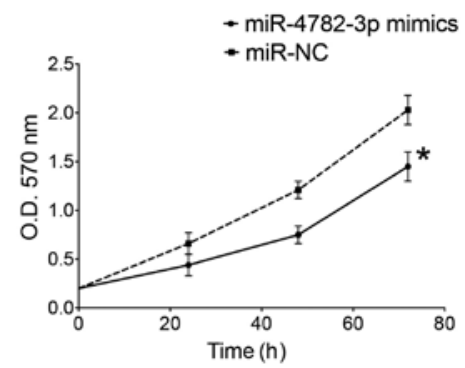

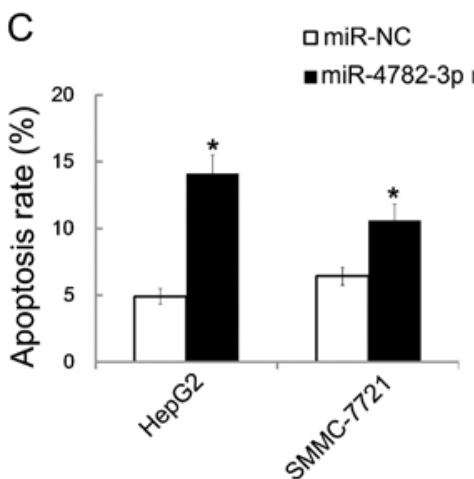

Figure 2. The effect of overexpression of miR-4782-3p on cell growth and apoptosis in vitro. After transfection of miR-4782-3p mimics, the level of miR-4782-3p in HepG-2 and SMMC-7721 cells were examined. The miR-4782-3p level in miR-NC mimics transfected cells was considered as $100 \%$ (A). The cell growth was assayed by MTT analysis at the indicated time-point (B). Following miR-4782-3p mimic transfection, cells were washed for PI/Annexin V staining, the apoptosis rate was examined by FACS (C). All data are mean \pm SD of three separate experiments. ${ }^{*}<<0.05$.

for $15 \mathrm{~min}$. The apoptotic rates were calculated on a flow cytometer (30).

Target prediction. Bioinformatics methods were applied for the prediction of the targeted genes of miR-4782-3p. Moreover, the bioinformatic algorithms from TargetScanHuman were used (31-34).

Dual-luciferase assays. To assess and confirm whether miR4782-3p binds USP14 directly, a dual-luciferase assay was performed $(25,30)$. The 3'UTR of USP14 was amplified by using PCR from genomic DNA. The production was inserted downstream of USP14 3'UTR reporter plasmids (pRL-USP14; Biotech, Chengdu, China), and mutants of USP14 3'UTR were generated by Site-Directed Mutagenesis kit (Shanghai, China). Then the whole plasmid was confirmed by sequencing. Mutation in the miR-4782-3p binding site of the USP14 3'UTR was constructed by Shengong Company (Shengong, Chengdu,
China). The luciferase reporter containing mutant was also constructed. For luciferase assays, HepG2 cells were transfected with luciferase reporter plasmid along with miR-4782-3p mimics or negative control by using Lipofectamine 2000 (Invitrogen). Twenty-four hours after transfection, these cells were analyzed by using a Luciferase assay kit (Promega, Madison, WI, USA) (35).

Statistical analysis. Two-tailed Student's t-test was used to analyze the difference between two groups. ANOVA was used to analyze the difference of three groups. Kaplan-Meier analysis was employed to evaluate the overall survival of HCC patients. The correlation between miR-4782-3p and USP14 was performed by Spearman's correlation analysis. All statistical analysis was performed by SPSS version 19 . Values are expressed as the mean \pm SD from three tests. All values of $\mathrm{P}<0.05$ are marked with an asterisk in the figures, and was considered statistically significantly different. 

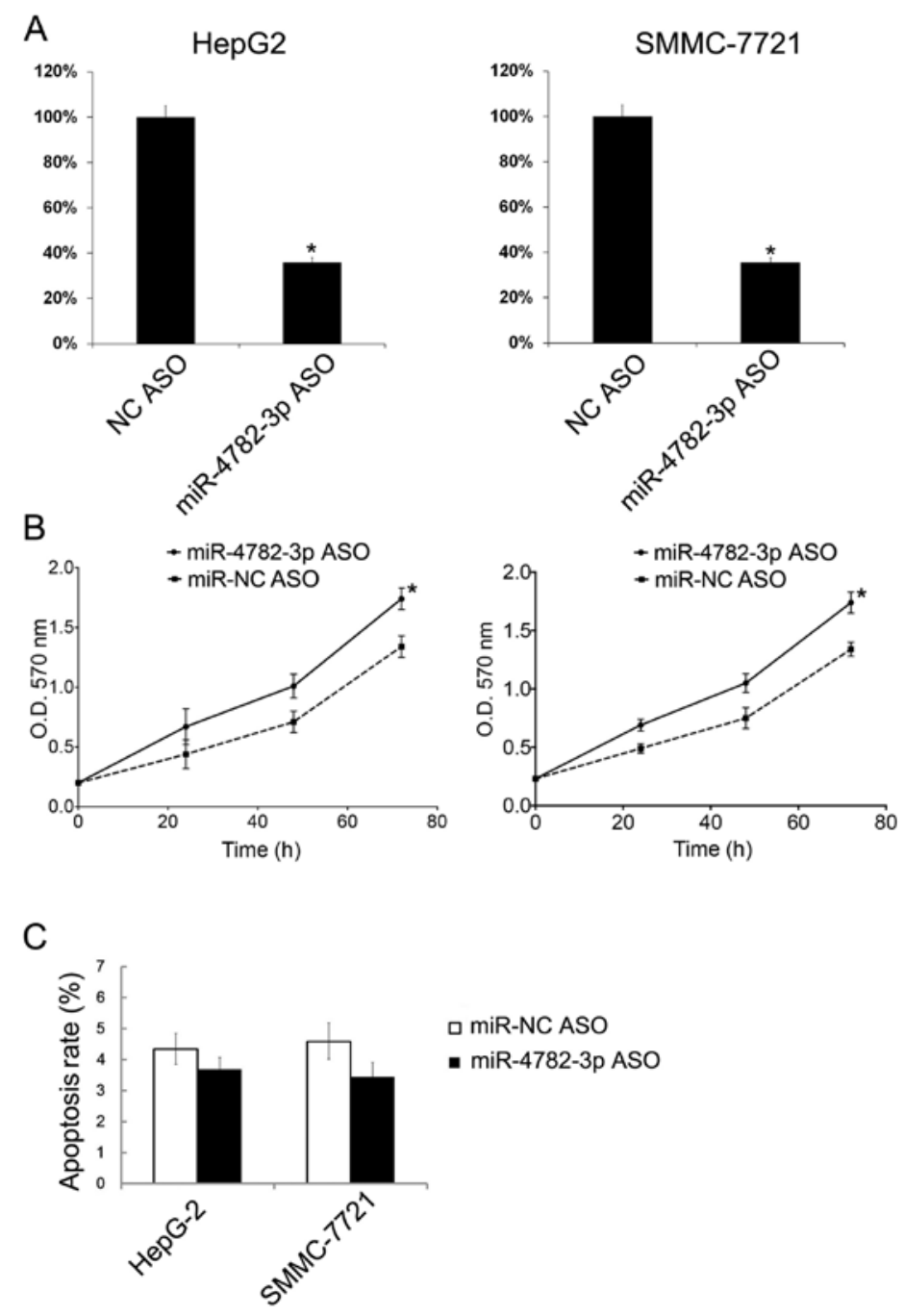

Figure 3. The effect of suppression of miR-4782-3p on cell growth and apoptosis in vitro. qRT-PCR analysis of miR-4782-3p in HepG-2 and SMMC-7721 cells upon transfection of miR-4782-3p ASO. U6 snRNA was used as negative control (A). MTT analysis showed that suppression of miR-4782-3p level in vitro promoted HepG-2 and SMMC-7721 cell growth (B). After miR-4782-3p ASO transfection into HepG-2 and SMMC-7721 cells, the cells were stained with $\mathrm{PI} /$ Annexin $\mathrm{V}$ for FACS analysis $(\mathrm{C})$. All data are mean $\pm \mathrm{SD}$ of three separate experiments. ${ }^{*} \mathrm{P}<0.05$.

\section{Results}

The low expression of miR-4782-3p in HCC tissues and its role in the survival of HCC. We first examined the miR-4782-3p level in HCC cell lines; the miR-4782-3p level in normal liver tissue was treated as a negative control. We found that the miR-4782-3p levels in HepG2 and SMMC-7721 cells were lower than in normal liver tissue and L-02 cells (Fig. 1A). Then we assayed the miR-4782-3p levels in 27 HCC tissues and adjacent normal liver tissues. We found that the mean value of miR-4782-3p levels in tumor tissues is lower than the mean value of adjacent normal tissues (Fig. 1B). The relative expression of miR-4782-3p in each HCC tissue and the matched adjacent normal liver tissue are shown in Fig. 1C. There were only 4 pairs, in which HCC tissues showed higher levels of miR-4782-3p. Next, the 27 HCC patients were separated into two groups by the median of the miR-4782-3p expression, and their progress was followed for about 100 weeks. We found that HCC patients with higher miR-4782-3p level had a longer survival period (Fig. 1D).
The effect of miR-4782-3p mimics on cells growth and apoptosis. To investigate the effect of miR-4782-3p in cell growth and apoptosis, HepG2 and SMMC-7721 cells were transiently transfected with miR-4782-3p mimics, and then the cell growth and apoptosis were evaluated. Forty-eight hours after transfection of the miR-4782-3p mimic, the miR4782-3p level in HepG2 and SMMC-7721 cells was tested by qRT-PCR. It showed that the miR-4782-3p levels in both cell types were increased (Fig. 2A). We then detected the effect of miR-4782-3p on cell growth by MTT. With transfection of miR-4782-3p mimic, HepG2 and SMMC-7721 cells showed significant decrease in cellular growth (Fig. 2B). To verify whether miR-4782-3p could influence HCC cells apoptosis, PI/ Annexin $\mathrm{V}$ double staining were performed to evaluate apoptosis. The results showed that $\mathrm{miR}-4782-3 \mathrm{p}$ mimic transfection increased apoptosis about 3-5\% (Fig. 2C).

The effect of miR-4782-3p ASO on cell growth and apoptosis. Additionally, HepG2 and SMMC-7721 cells were transfected with miR-4782-3p ASO to downregulate the miR-4782-3p 
Position 524-530 of USP14 3' UTR 5' ...CAUGGAAACAUGGgCACAAUCAA... hsa-miR-4782-3p 3 ' CAAGAUCUAUACUUCUGUUAGU

Mutated version of USP14 3' UTR 5 '...CAUGGAAACAUGGGCAAAAAAAA...

B

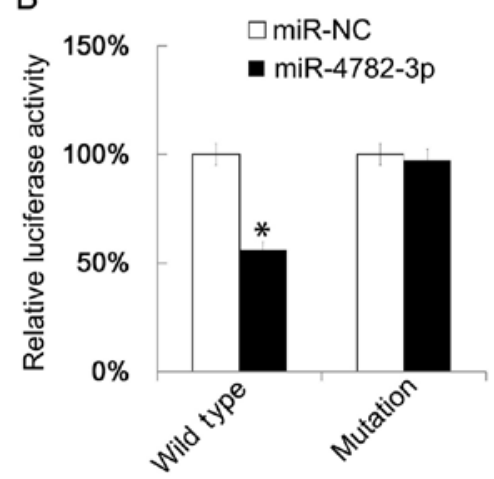

C

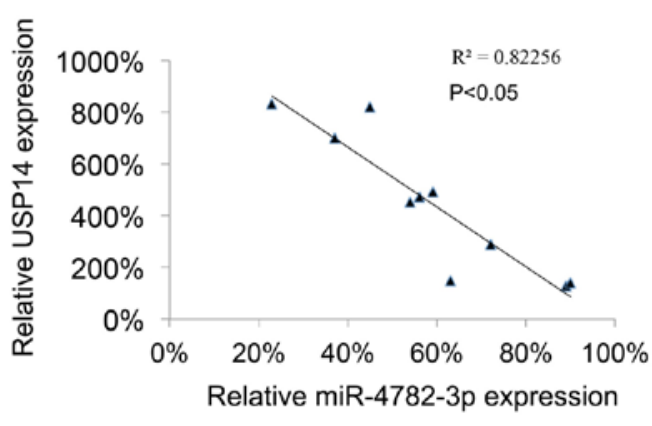

Figure 4. USP14 is targeted by miR-4782-3p. TargetScanHuman (www. targetscan. org) showed that USP14 was a direct target of miR-4782-3p (A). HepG-2 cells were co-transfected with miR-4782-3p mimics or control and reporter plasmid or the mutant 3'UTR of USP14, together with the controls. Thirty-six hours after transfection, the luciferase activity was measured (B). USP14 mRNA expressions in 10 HCC tissues were examined by qRT-PCR. The levels of miR-4782-3p and USP14 in HCC tissues were negatively correlated (C). All value are mean \pm SD of three separate experiments. * $\mathrm{P}<0.05$.

levels. Then the miR-4782-3p ASO transfection was tested by qRT-PCR. The miR-4782-3p levels in both cell lines decreased following the transfection (Fig. 3A). With miR-4782-3p ASO transfection, HepG2 and SMMC-7721 cells showed significant increase in cellular growth (Fig. 3B). However, the miR4782-3p ASO showed no significant effect on cell apoptosis (Fig. 3C).

miR-4782-3p targets USP14. The gene targets of miR-4782-3p were predicted by TargetScanHuman, this prediction showed that USP14 could be targeted by miR-4782-3p. Then we mutated the binding site of miR-4782-3p in USP14 (Fig. 4A). The effect of miR-4782-3p on USP14 translation was tested by a luciferase reporter assay. The luciferase reporter plasmid with wild-type 3'UTR of USP14 or the mutated version as indicated in Fig. 4A showed that upregulation of miR-4782-3p reduced the luciferase activity of the reporter gene with wildtype, but not with the mutant (Fig. 4B). Next the USP14 mRNA expressions in 10 HCC tissues were examined by qRT-PCR. We found that the miR-4782-3p level and the USP14 mRNA level were negatively correlated (Fig. 4C).

\section{Discussion}

miR-4782-3p has shown its important role in the pathogenesis of NSCLC. miR-4782-3p in NSCLC tissues was relatively low. High expression of miR-4782-3p indicated favorable prognosis of NSCLC patients. The targeted genes were USP14, ZEB2 and XIAP (25). In this study, the role of miR-4782-3p in HCC was studied. We demonstrated miR-4782-3p also showed a low level in HCC tissues. Importantly, HCC patients with higher miR-4782-3p level had longer survival period. The underlying mechanism may be that the level of miR-4782-3p in HCC promoted cell growth and inhibited apoptosis. To the best of our knowledge, this is the first report on the role of miR-4782-3p in HCC.

USP14 has been proven to be the target gene of miR-4782-3p, and another study showed that USP14 activation promotes tumor progression in HCC (26). Thus our study has established a connection between miR-4782-3p and USP14 in HCC.

USP14 plays important roles in different types of cancers. For example, overexpression of USP14 in NSCLC was associated with shorter overall survival of patients (36). High expression of USP14 was related to poor prognosis of epithelial ovarian cancer patients (37). Thus an inhibitor of USP14, like miR-4782-3p, may have a potential therapeutic effect.

Our data showed that overexpression of miR-4782-3p could increase the apoptosis rate of HepG-2 and SMMC-7721 cells, however, apoptosis of these cell lines did not decrease significantly after downregulation of miR-4782-3p. We considered the reason to be that the apoptosis rate of HepG-2 and SMMC-7721 is very low, thus it is hard to reduce it to even lower apoptosis rate.

In conclusion, this study revealed the role of miR-4782-3p in HCC. Our study contributes to finding a new target for HCC therapy.

\section{References}

1. Kamangar F, Dores GM and Anderson WF: Patterns of cancer incidence, mortality, and prevalence across five continents: Defining priorities to reduce cancer disparities in different geographic regions of the world. J Clin Oncol 24: 2137-2150, 2006.

2. Torre LA, Bray F, Siegel RL, Ferlay J, Lortet-Tieulent J and Jemal A: Global cancer statistics, 2012. CA Cancer J Clin 65: 87-108, 2015. 
3. El-Serag HB: Epidemiology of viral hepatitis and hepatocellular carcinoma. Gastroenterology 142: 1264-1273.e1, 2012.

4. Gao J, Xie L, Yang WS, Zhang W, Gao S, Wang J and Xiang YB: Risk factors of hepatocellular carcinoma - current status and perspectives. Asian Pac J Cancer Prev 13: 743-752, 2012.

5. Portolani N, Coniglio A, Ghidoni S, Giovanelli M, Benetti A, Tiberio GA and Giulini SM: Early and late recurrence after liver resection for hepatocellular carcinoma: Prognostic and therapeutic implications. Ann Surg 243: 229-235, 2006.

6. Gramantieri L, Ferracin M, Fornari F, Veronese A, Sabbioni S, Liu CG, Calin GA, Giovannini C, Ferrazzi E, Grazi GL, et al: Cyclin G1 is a target of miR-122a, a microRNA frequently down-regulated in human hepatocellular carcinoma. Cancer Res 67: 6092-6099, 2007.

7. Budhu A, Jia HL, Forgues M, Liu CG, Goldstein D, Lam A, Zanetti KA, Ye QH, Qin LX, Croce CM, et al: Identification of metastasis-related microRNAs in hepatocellular carcinoma Hepatology 47: 897-907, 2008.

8. Xu T, Zhu Y, Xiong Y, Ge YY, Yun JP and Zhuang SM: MicroR NA-195 suppresses tumorigenicity and regulates G1/S transition of human hepatocellular carcinoma cells. Hepatology 50: 113-121, 2009.

9. Su H, Yang JR, Xu T, Huang J, Xu L, Yuan Y and Zhuang SM: MicroRNA-101, down-regulated in hepatocellular carcinoma, promotes apoptosis and suppresses tumorigenicity. Cancer Res 69: 1135-1142, 2009.

10. Murakami Y, Yasuda T, Saigo K, Urashima T, Toyoda H, Okanoue $\mathrm{T}$ and Shimotohno K: Comprehensive analysis of microRNA expression patterns in hepatocellular carcinoma and non-tumorous tissues. Oncogene 25: 2537-2545, 2006.

11. Braconi $\mathrm{C}$ and Patel T: MicroRNA expression profiling: A molecular tool for defining the phenotype of hepatocellular tumors. Hepatology 47: 1807-1809, 2008.

12. Murakami Y, Tamori A, Itami S, Tanahashi T, Toyoda $\mathrm{H}$ Tanaka M, Wu W, Brojigin N, Kaneoka Y, Maeda A, et al: The expression level of miR-18b in hepatocellular carcinoma is associated with the grade of malignancy and prognosis. BMC Cancer 13: 99, 2013.

13. Gramantieri L, Fornari F, Callegari E, Sabbioni S, Lanza G, Croce CM, Bolondi L and Negrini M: MicroRNA involvement in hepatocellular carcinoma. J Cell Mol Med 12: 2189-2204, 2008.

14. Wang B, Majumder S, Nuovo G, Kutay H, Volinia S, Patel T, Schmittgen TD, Croce C, Ghoshal K and Jacob ST: Role of microRNA-155 at early stages of hepatocarcinogenesis induced by choline-deficient and amino acid-defined diet in C57BL/6 mice. Hepatology 50: 1152-1161, 2009.

15. Coulouarn C, Factor VM, Andersen JB, Durkin ME and Thorgeirsson SS: Loss of miR-122 expression in liver cancer correlates with suppression of the hepatic phenotype and gain of metastatic properties. Oncogene 28: 3526-3536, 2009.

16. Ji J, Shi J, Budhu A, Yu Z, Forgues M, Roessler S, Ambs S, Chen Y, Meltzer PS, Croce CM, et al: MicroRNA expression, survival, and response to interferon in liver cancer. N Engl J Med 361: 1437-1447, 2009.

17. Ji J, Yamashita T, Budhu A, Forgues M, Jia HL, Li C, Deng C, Wauthier E, Reid LM, Ye QH, et al: Identification of microRNA-181 by genome-wide screening as a critical player in EpCAM-positive hepatic cancer stem cells. Hepatology 50: 472-480, 2009

18. Ladeiro Y, Couchy G, Balabaud C, Bioulac-Sage P, Pelletier L, Rebouissou $\mathrm{S}$ and Zucman-Rossi J: MicroRNA profiling in hepatocellular tumors is associated with clinical features and oncogene/tumor suppressor gene mutations. Hepatology 47: 1955-1963, 2008.

19. Song G, Sharma AD, Roll GR, Ng R, Lee AY, Blelloch RH, Frandsen NM and Willenbring H: MicroRNAs control hepatocyte proliferation during liver regeneration. Hepatology 51 1735-1743, 2010.
20. Ura S, Honda M, Yamashita T, Ueda T, Takatori H, Nishino R, Sunakozaka H, Sakai Y, Horimoto K and Kaneko S: Differential microRNA expression between hepatitis B and hepatitis Cleading disease progression to hepatocellular carcinoma. Hepatology 49 : 1098-1112, 2009

21. Wong TS, Liu XB, Wong BY, Ng RW, Yuen AP and Wei WI Mature miR-184 as potential oncogenic microRNA of squamous cell carcinoma of tongue. Clin Cancer Res 14: 2588-2592, 2008.

22. Wang Y, Lee AT, Ma JZ, Wang J, Ren J, Yang Y, Tantoso E, Li KB, Ooi LL, Tan P, et al: Profiling microRNA expression in hepatocellular carcinoma reveals microRNA-224 up-regulation and apoptosis inhibitor-5 as a microRNA-224-specific target. J Biol Chem 283: 13205-13215, 2008.

23. Yuan B, Liang Y, Wang D and Luo F: miR-940 inhibits hepatocellular carcinoma growth and correlates with prognosis of hepatocellular carcinoma patients. Cancer Sci 106: 819-824, 2015.

24. Li D, Liu X, Lin L, Hou J, Li N, Wang C, Wang P, Zhang Q, Zhang P, Zhou W, et al: MicroRNA-99a inhibits hepatocellular carcinoma growth and correlates with prognosis of patients with hepatocellular carcinoma. J Biol Chem 286: 36677-36685, 2011.

25. Wu N, Zhang C, Bai C, Han YP and Li Q: miR-4782-3p inhibited non-small cell lung cancer growth via USP14. Cell Physiol Biochem 33: 457-467, 2014

26. Huang G, Li L and Zhou W: USP14 activation promotes tumor progression in hepatocellular carcinoma. Oncol Rep 34: 2917-2924, 2015

27. Song B, Zhang C, Li G, Jin G and Liu C: miR-940 inhibited pancreatic ductal adenocarcinoma growth by targeting MyD88. Cell Physiol Biochem 35: 1167-1177, 2015.

28. Liu C, Gao F, Li B, Mitchel RE, Liu X, Lin J, Zhao L and Cai J: TLR4 knockout protects mice from radiation-induced thymic lymphoma by downregulation of IL6 and miR-21. Leukemia 25 : 1516-1519, 2011.

29. Liu C, Zhou C, Gao F, Cai S, Zhang C, Zhao L, Zhao F, Cao F, Lin J, Yang Y, et al: miR-34a in age and tissue related radio-sensitivity and serum miR-34a as a novel indicator of radiation injury. Int J Biol Sci 7: 221-233, 2011.

30. Lin L, Liang H, Wang Y, Yin X, Hu Y, Huang J, Ren T, Xu H, Zheng $L$ and Chen X: microRNA-141 inhibits cell proliferation and invasion and promotes apoptosis by targeting hepatocyte nuclear factor- $3 \beta$ in hepatocellular carcinoma cells. BMC Cancer 14: 879, 2014.

31. Lewis BP, Burge CB and Bartel DP: Conserved seed pairing, often flanked by adenosines, indicates that thousands of human genes are microRNA targets. Cell 120: 15-20, 2005.

32. Friedman RC, Farh KK, Burge CB and Bartel DP: Most mammalian mRNAs are conserved targets of microRNAs. Genome Res 19: 92-105, 2009.

33. Grimson A, Farh KK, Johnston WK, Garrett-Engele P, Lim LP and Bartel DP: MicroRNA targeting specificity in mammals: Determinants beyond seed pairing. Mol Cell 27: 91-105, 2007.

34. Garcia DM, Baek D, Shin C, Bell GW, Grimson A and Bartel DP: Weak seed-pairing stability and high target-site abundance decrease the proficiency of 1sy- 6 and other microRNAs. Nat Struct Mol Biol 18: 1139-1146, 2011.

35. Grentzmann G, Ingram JA, Kelly PJ, Gesteland RF and Atkins JF: A dual-luciferase reporter system for studying recoding signals. RNA 4: 479-486, 1998.

36. Wu N, Liu C, Bai C, Han YP, Cho WC and Li Q: Over-expression of deubiquitinating enzyme USP14 in lung adenocarcinoma promotes proliferation through the accumulation of $\beta$-catenin. Int J Mol Sci 14: 10749-10760, 2013.

37. Wang Y, Wang J, Zhong J, Deng Y, Xi Q, He S, Yang S, Jiang L, Huang M, Tang C, et al: Ubiquitin-specific protease 14 (USP14) regulates cellular proliferation and apoptosis in epithelial ovarian cancer. Med Oncol 32: 379, 2015. 